\title{
The study of Jak2 V617F mutation in polycythemia vera with zebrafish model
}

\author{
Alvin $\mathrm{CH} \mathrm{Ma}{ }^{1}$, Alice MS Cheung ${ }^{1}$, Alister C Ward ${ }^{1}$, Wing-Yan Au ${ }^{1}$, Yok-Lam Kwong ${ }^{1}$, Raymond Liang ${ }^{1}$, Anskar \\ YH Leung ${ }^{1}$ \\ ${ }^{1}$ Department of Medicine, The University of Hong Kong, Hong Kong, China
}

Polycythemia vera (PV) is a myeloproliferative disease resulting from clonal proliferation of hematopoietic stem cells (HSC). In most PV patients, the substitution of valine by phenylalanine at amino acid 617 in the autoregulatory $\mathrm{JH} 2$ domain $(\mathrm{V} 617 \mathrm{~F})$ results in constitutive activation of $\mathrm{Jak2}\left(\mathrm{Jak2}^{\mathrm{V} 617 \mathrm{~F}}\right)$, conferring proliferative and survival advantage to erythroid progenitors. However, the impact of such mutation on HSC is not fully understood. This is now examined using the zebrafish models. In zebrafish, the gene encoding for Jak2 has undergone duplication and subsequent specialization into jak2a and jak2b. Previous studies have demonstrated that jak2a play an important role in zebrafish primitive hematopoiesis via the Jak-Stat pathway. In these study, a zebrafish jak2a ${ }^{\mathrm{V} 81 \mathrm{~F}}$ mutant clone, corresponding to human $\mathrm{Jak}^{\mathrm{V}}{ }^{\mathrm{V} 17 \mathrm{~F}}$, was generated by site-directed mutagenesis. The mutant clone was in vitro transcribed into mRNA and injected into early 1-cell stage zebrafish embryos. There was no morphological abnormality in the injected embryos up to 48 hour-post-fertilization (hpf) and the effects on erythroid and stem cell populations were examined using flow cytometry and quantitative reverse-transcriptase PCR. By flow cytometry, expression of jak2a ${ }^{\mathrm{V} 581 \mathrm{~F}}$ in zebrafish embryos significantly increased the percentage of gata-1 expressing erythroids at $18 \mathrm{hpf}$ and could be rescued through co-injecting with stat5a morpholino. On the other hand, quantitative PCR shown that at the same stage, the expression of genes associated with erythroid including gata-1, $\alpha$ - and $\beta$ - embryonic hemoglobins but not stem cell lineage likes scl or $\mathbf{l m o 2}$ were affected. Western blotting analysis confirmed that the V581F mutation acts in a Stat5-phosporylation dependent fashion. Together, these observations supported a conserved paradigm in zebrafish and human PV in which Jak2 mutation affects lineage-committed rather than stem cell population.

Keywords: polycythemia vera, myeloproliferative disease, Jak2 ${ }^{\mathrm{V} 617 \mathrm{~F}}$ mutation, zebrafish

Cell Research (2008) 18:s141. doi: 10.1038/cr.2008.231; published online 4 August 2008

Correspondence: Alvin CH MA

E-mail: h0025231@hkusua.hku.hk 